\title{
A young woman with palpitations, goitre and low thyroid- stimulating hormone
}

\author{
Henry B. Burch MD, David S. Cooper MD
}

\begin{abstract}
A 23-year-old woman reports palpitations, difficulty sleeping, heat intolerance and weight loss over a two-month period. Evaluation shows a pulse of 110 beats/min; blood pressure 135/70 mm Hg; normal eye examination; an enlarged, nontender thyroid; and tremor. Her serum thyroid-stimulating hormone (TSH) level is less than 0.01 (normal 0.4-4.2) $\mathrm{mlU} / \mathrm{L}$.
\end{abstract}

\section{Should this patient go to the emergency department?}

She is hemodynamically stable and has no features of thyroid storm, such as fever, changes to mental status, tachyarrhythmia, congestive heart failure or hepatic dysfunction. ${ }^{1,2}$ She requires timely evaluation and treatment, but not an urgent referral to the emergency department. Conversely, older patients with severe hyperthyroidism and cardiovascular decompensation require urgent intervention.

\section{What further investigations are needed?}

In this patient with typical symptoms and signs of thyrotoxicosis, and an enlarged thyroid gland, the differential diagnosis aside from thyroid disease is limited. Box 1 lists common and uncommon causes of thyrotoxicosis. In this patient, given the absence of eye findings and the diffuse, nontender goitre, the two diagnoses that are most likely are Graves disease and painless thyroiditis. Either radioactive iodine uptake (RAIU) or TSH receptor antibody testing should be done to establish the correct diagnosis. Current guidelines favour the RAIU test as the preferred diagnostic tool to distinguish Graves disease from painless thyroiditis. ${ }^{3}$ If the diagnosis is obviously Graves disease, such as in a patient with diffuse goitre with a bruit and ophthalmopathy, thyroid scanning to look at thyroid morphology or RAIU is not required to confirm the diagnosis. Likewise, TSH receptor antibody testing is generally not needed to make the diagnosis in a classic case of Graves disease, but it may be useful in establishing the etiology of thyrotoxicosis for patients in whom diagnostic uncertainty exists.

Pregnancy status should be established in women of childbearing age, because this affects both the choice of therapy and surveillance tempo. Patients being considered for treatment with antithyroid drugs should have a baseline complete blood count and liver enzyme testing.

\section{What is the interim management for this patient?}

$\beta$-adrenergic blocking agents are an important adjunctive therapy in patients with palpitations, tremor and nervousness, as seen in this patient. Guidelines recommend consideration of these agents in all symptomatic patients. ${ }^{3}$ Long-acting drugs such as atenolol, nadolol, or long-acting propranolol or metoprolol are preferred over shorter-acting agents. ${ }^{3} \beta$-blockers are generally well-tolerated but can lead to exacerbation of asthma and worsening congestive heart failure; they are contraindicated in patients with Raynaud phenomenon.

\section{What definitive management should be considered?}

For definitive management, the three traditional treatments for hyperthyroidism caused by Graves disease are an antithyroid drug, ${ }^{4}$ radioiodine ${ }^{5}$ and surgery (Box 2). Current guidelines recommend methimazole as the preferred antithyroid drug, because of the potential for severe hepatotoxicity associated with propylthiouracil. ${ }^{3}$ In the first trimester of pregnancy, however, propylthiouracil is recommended because of possible teratogenic effects linked to methimazole. ${ }^{3}$ Patients experiencing minor adverse drug reactions to methimazole (e.g., fever, rash, arthralgia, agranulocytosis and jaundice) who are not candidates for radioiodine or surgery may also be given propylthiouracil.

The choice of therapy for Graves disease is based on a variety of clinical factors and per-
Competing interests: Both authors served on the task force for the American Thyroid Association guidelines cited in this paper.

This article has been peer reviewed.

Correspondence to:

David S. Cooper,

dscooper@jhmi.edu

CMAJ 2014. DOI:10.1503 /cmaj.130558 
sonal preferences of the patient. ${ }^{3}$ For example, certain clinical factors, such as mild hyperthyroidism and a small goitre, are associated with a higher likelihood of achieving a remission on antithyroid drugs. Likewise, a patient who wishes to avoid lifelong therapy with thyroid hormone may select a trial of medical treatment rather than surgery or radioactive iodine therapy.

Whether a patient should be given methimazole pending a consultation with an endocrinologist depends on the time it takes for the appointment, the severity of the patient's symptoms, and the degree of his or her thyroid dysfunction (serum free thyroxine $\left[\mathrm{FT}_{4}\right]$, triiodothyronine $\left[\mathrm{T}_{3}\right]$ or free $\mathrm{T}_{3}$ level $>3$ or 4 times the upper limit of normal is considered severe), as well as the

\section{Box 1: Causes of thyrotoxicosis ${ }^{3}$ \\ Overproduction of thyroid hormone \\ Common \\ - Graves disease \\ - Toxic multinodular goitre \\ - Solitary toxic adenoma \\ Less common \\ - lodine-induced \\ - Gestational thyrotoxicosis (hCG-mediated) \\ Rare \\ - Amiodarone-induced (type 1$)^{*}$ \\ - Choriocarcinoma (hCG-mediated)}

Release of thyroid from damaged gland Common

- Postpartum thyroiditis

- Painless thyroiditis

Less common

- Subacute thyroiditis

- Amiodarone-induced (type 2)

Rare

- Radiation thyroiditis

Exogenous thyroid hormone excess

Common

- latrogenic (excessive thyroid hormone therapy)

Less common

- Dietary supplements

Rare

- Surreptitious use of thyroid hormone

- Hamburger thyrotoxicosis

- Accidental ingestion of thyroxine $\left(\mathrm{T}_{4}\right)$ or triiodothyronine $\left(T_{3}\right)$

Note: $\mathrm{hCG}=$ human chorionic gonadotropin.

*More common in Europe than in North America, because patients living in a relatively iodine-deficient environment are more susceptible to iodine-induced hyperthyroidism (the so-called Jod-Basedow phenomenon). provider's familiarity with the drug. Methimazole should be taken for at least one year to maximize the likelihood of remission. ${ }^{4}$

\section{The case revisited}

Results of thyroid function tests were as follows: $\mathrm{FT}_{4} 32.2$ (normal 10.3-19.6) pmol/L, T 4.62 (normal 1.2-3.1) nmol/L and repeat TSH was again less than $0.01 \mathrm{mIU} / \mathrm{L}$. The patient was prescribed atenolol $50 \mathrm{mg}$ daily, and her symptoms improved. She saw an endocrinologist three weeks later. The patient opted for treatment with methimazole and was prescribed $15 \mathrm{mg} / \mathrm{d}$ after testing of her baseline thyroid function, complete blood

\section{Box 2: Treatment of Graves disease ${ }^{3-5}$}

\section{Antithyroid drugs}

Advantages

- Nonablative

Frequency of hypothyroidism

- Low frequency

Other disadvantages

- Not a definitive treatment

- Adverse effects

Cost

- Lowest cost

\section{Radioiodine treatment}

Advantages

- Definitive

Frequency of hypothyroidism

- All (100\%)

Other disadvantages

- Patient fear

- Worsening of eye disease, likely related to an increase in TSH receptor antibody levels*

Cost

- Higher cost

\section{Surgery}

Advantages

- Definitive

Frequency of hypothyroidism

- All (100\%)

Other disadvantages

- Complications related to surgery

- Discomfort

Cost

- Highest cost

Note: TSH = thyroid-stimulating hormone.

*Theoretically, this could create problems in women who wish to become pregnant in the future, because TSH

receptor antibodies can cross the placenta and rarely lead

to neonatal thyroid dysfunction. However, there are no

data to specifically address this issue.

tOver time, radioiodine may be the most cost-effective therapy. 
count and liver enzyme levels. She was also provided with information about adverse effects related to treatment with methimazole.

The patient's thyroid function returned to normal six weeks later, and the methimazole dose was decreased to $10 \mathrm{mg} / \mathrm{d}$ and then to $5 \mathrm{mg} / \mathrm{d}$ over the next three months. She was instructed to stop taking the drug after one year of treatment, and testing of her thyroid function remained normal over the next year. The patient was advised to have annual testing of her thyroid function and to avoid smoking, because smoking increases the risk of relapse. ${ }^{6}$ She was told that she should have TSH receptor antibody testing performed during pregnancy to assess the risk for fetal thyroid dysfunction and that Graves disease is likely to relapse after pregnancy. ${ }^{7}$

\section{References}

1. Akamizu T, Satoh T, Isozaki O, et al. Diagnostic criteria, clinical features and incidence of thyroid storm based on nationwide surveys. Thyroid 2012;22:661-79.

2. Burch HB, Wartofsky L. Life-threatening thyrotoxicosis: thyroid storm. Endocrinol Metab Clin North Am 1993;22:263-77.

3. Bahn RS, Burch HB, Cooper DS, et al. Hyperthyroidism and other causes of thyrotoxicosis: management guidelines of the American Thyroid Association and American Association of Clinical Endocrinologists. Thyroid 2011;21:593-646.

4. Cooper DS. Antithyroid drugs. N Engl J Med 2005;352:905-17.
5. Ross DS. Radioiodine therapy for hyperthyroidism. $N$ Engl J Med 2011;364:542-550

6. Quadbeck B, Roggenbuck U, Janssen OE, et al.; Basedow Study Group. Impact of smoking on the course of Graves' disease after withdrawal of antithyroid drugs. Exp Clin Endocrinol Diabetes 2006;114:406-11.

7. Stagnaro-Green A, Abalovich M, Alexander E, et al.; American Thyroid Association Taskforce on Thyroid Disease During Pregnancy and Postpartum. Guidelines of the American Thyroid Association for the diagnosis and management of thyroid disease during pregnancy and postpartum. Thyroid 2011;21:1081-125.

Affiliations: Division of Endocrinology, Diabetes, and Metabolism (Burch), Walter Reed National Military Medical Center, Bethesda, Md.; Division of Endocrinology, Diabetes, and Metabolism (Cooper), The Johns Hopkins University School of Medicine, Baltimore, Md.

Contributors: Both authors wrote and revised the article, and approved the final version submitted for publication.

Decisions is a series that focuses on practical evidence-based approaches to common presentations in primary care. The articles address key decisions that a clinician may encounter during initial assessment. The information presented can usually be covered in a typical primary care appointment. Articles should be no longer than 650 words, may include one box, figure or table and should begin with a very brief description (75 words or less) of the clinical situation. The decisions addressed should be presented in the form of questions. A box providing helpful resources for the patient or physician is encouraged.

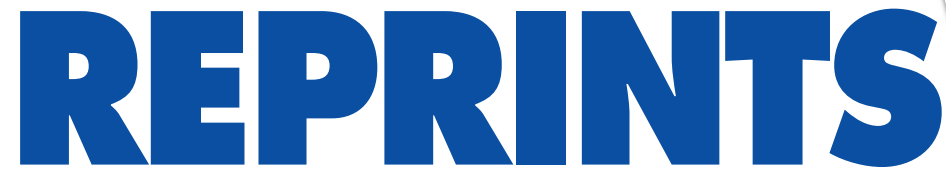

We have partnered with Sheridan Press!

To purchase commercial article reprints and e-prints or to request a quote, please contact

Matt Neiderer

Content Sales Sheridan Content Services
$800635-7181 \times 8265$ matt.neiderer@sheridan.com 